\title{
Inter-cell Interference Management Technique for Multi-Cell LTE-A Network
}

\author{
Iskandar, I. Setyawan, and H. Nuraini \\ School of Electrical Engineering and Informatics, Bandung Institute of Technology, Indonesia
}

\begin{tabular}{|c|c|}
\hline Article Info & ABSTRACT \\
\hline Article history: & In modern cellular system such as LTE Advanced (LTE-A), frequency reuse \\
\hline Received Mar 14, 2017 & $\begin{array}{l}\text { scheme is targeted to be applied to fulfill the requirement of high capacity } \\
\text { broadband access and high spectrum efficiency. But this kind of frequency }\end{array}$ \\
\hline Revised Jun 5, 2017 & planning may lead to the worse inter-cell interference (ICI) level experienced \\
\hline Accepted Aug 11, 2017 & $\begin{array}{l}\text { especially by a user located at the cell edge. Soft Frequency Reuse (SFR) is } \\
\text { considered as an effective way to mitigate inter-cell interference and }\end{array}$ \\
\hline Keywords: & $\begin{array}{l}\text { maintain capacity. We propose a power division SFR, known as multi level } \\
\text { SFR technique to minimize ICI in a designed LTE-A network for sub-urban }\end{array}$ \\
\hline BLER & environment. Service area of LTE-A network was first developed to deploy \\
\hline CIR downlink & particular number of eNB by using LTE network planning tools in the \\
\hline Dimensioning & $\begin{array}{l}\text { frequency of } 1800 \mathrm{MHz} \text { with the use of SISO (Single Input Single Output) } \\
\text { antennas. Coverage dimensioning and propagation consideration determine }\end{array}$ \\
\hline LTE & LTE-A parameters which were used in the simulation. Monte carlo \\
\hline SINR & simulation is executed to examine the performance of SFR for LTE-A \\
\hline Soft frequency reuse (SFR) & downlink transmission to address different power ratio and traffic loads \\
\hline Throughput & $\begin{array}{l}\text { problem. Both performance of cell edge users and overall cell performance } \\
\text { are evaluated in terms of CINR, BLER, and throughput. Performance with } \\
\text { SFR is also compared with the classical frequency reuse one and three. }\end{array}$ \\
\hline
\end{tabular}

Copyright @ 2017 Institute of Advanced Engineering and Science. All rights reserved.

\section{Corresponding Author:}

Iskandar,

School of Electrical Engineering and Informatics,

Bandung Institute of Technology,

Jalan Ganesha No. 10, Bandung 40132, Indonesia.

Email: iskandar@stei.itb.ac.id

\section{INTRODUCTION}

The large growing of data transactions over new-generation smart phones and mobile internet devices has lead an essential need for an efficient network [1]. High-bandwidth consumption services such as video streaming and mobile cloud have caused exponential increased of data traffic. Therefore an efficient cellular network is required to provide such services. LTE-A is the prominent solution mobile cellular technology that combine the conventional high power macro cell base station (eNB) overlaid with low power cell such micro and pico cells. In such heterogeneous multi-cells network, inter-cell interference can not be avoided and even become a big problem that could bring the capacity and spectrum efficiency become worse.

One solution to maximize the efficiency of the network is to minimize interference which has been a major obstacle for the network designer. Broadly speaking, the interference is divided into two types, namely co-channel interference and adjacent interference. Adjacent interference is the interference that occurs at the end of the frequency band in each cell. The interference can cause a decrease in network capacity and coverage, but can be minimized by using proper frequency planning in adjacent cells. The co-channel interference occurs due to the same frequency band in different cell reuse. To minimize the co-channel interference, the use of cell sectorization has been applied, in which each sector antenna covers a particular region of the cell. On the use of three sector antennas, each antenna covers 120 degrees of part of the cell cycle, and can reach a considerable distance from the antenna site. In addition, also known as the antenna 6 
sector, which covers 60 degrees of the parts of the cell. This system is also called frequency reuse system, wherein a channel bandwidth is broken down by the number of sectors in the cell, and the same channel used for the other sectors at a given distance from the cell. The emerging challenges in the use of frequency reuse is increasingly smaller network capacity in each cell, so that each customer throughput becomes smaller. This of course is very detrimental, given the need for greater network capacity more is needed to be able to enjoy various services provided in the mobile technology-based broadband. For that, we need an effective method to minimize interference on cells without reducing the cell capacity.

\section{SOFT FREQUENCY REUSE (SFR) AND PROBLEM STATEMENT}

In cellular system, soft frequency reuse (SFR) has become one of the most important enablers especially in 3G and 4G LTE cellular system [2]-[7]. In 3GPP LTE system, to achieve high data-rate transmission, it has been extensively explored under the subject of inter cell interference coordination (ICIC). In this paper, simulation design of LTE-A network is developed to study the performance of SFR using power division method. As we may know that in SFR, for each cell there has been a limitation for a power density upper limit (PDL) curve [8]. The actual power density of the transmitted signal should be lower than the PDL. In this concept, the whole bandwidth is divided into three parts, namely $\mathrm{f} 1, \mathrm{f} 2$, and $\mathrm{f} 3$ as shown in Figure 1. In each cell, one part, called primary band, is configured to have a higher PDL than the other two, called secondary bands. SFR is considered as one of the most effective frequency planning methods as an Inter-Cell Interference Coordination (ICIC) in the current mobile cellular system. SFR was initially introduced in the GSM system, and later is adopted into the 3GPP LTE with the objective of providing better performance for customers who are on the border of the cell.

Figure 1 shows SFR scheme that is applied to the seven cells. The basic idea of the scheme is to implement the SFR with reuse factor equals 1 (FR1) for the cell-center users (CCU) and reuse factor equals 3 (FR3) for cell-edge users (CEU) [9]. One-third of the entire bandwidth or well known as a major segment can be used for CEU, through which packets are sent with greater power. In order to qualify for CEU FR3, major segment must be mutually orthogonal with the CEU on neighboring cells. Meanwhile, the CCU can access the entire frequency, with lower transmission power, assuming a total power equal to the FR1 is transmitted for the SFR power, and that power is distributed evenly across physical resource block (PRB). Therefore, in FR1, power per PRB is $P=P_{\text {Total }} / N$, where $P_{\text {Total }}$ is the total transmitted power and $\mathrm{N}$ is the number of total PRB available in the cell. In SFR, if power per PRB is defined as the $P_{C E U}$, then the power per PRB in the middle of the cell (PCCU) is $\alpha P_{C E U}$, where $\alpha$ is defined as the power ratio between the two power $\left(P_{C C U} / P_{C E U}\right) . P_{C E U}$ can be obtained through the Equation 1 and 2.

$$
\begin{aligned}
& P_{C E U} \frac{1}{3} N+\alpha P_{C E U} \frac{2}{3} N=P_{\text {Total }} \\
& P_{C E U}=\frac{3 P_{\text {Total }}}{N(1+\alpha)}
\end{aligned}
$$

By looking at the design of SFR, some restrictions apply. One thing that is common in cellular systems is the number of CEU more than CCU, considering the surface area that is in the outer area wider than part in it. However, in the scheme of this SFR, cell edge area is defined as the area of the outer third of a cell and the cell edge user can only use a maximum of $1 / 3$ of the total bandwidth (PRB). In this paper we study the implementation of SFR using power partition technique in the developed LTE-A network by using network tool simulator. The digital map of an area in which LTE-A was being deployed is used as an experimental area of observation. We use LTE-A frequency of $1800 \mathrm{MHz}$ and monte carlo simulation to observe the performance of SFR in the downlink transmission at the various transmission power and different traffic load. We also examine the performance of user at the cell edge as well as the average performance of the users in the entire cell by using CINR, BLER, and throughput parameter.

We consider an LTE-A system consists of $\mathrm{N}$ cells, where $\mathrm{N}$ is derived from the cell planning and network dimensioning based on LTE-A technical specification that employ for the particular urband and suburban area [11]. It also operates in Frequency-Division Duplex (FDD) mode, where we are interested in the downlink transmission with a number of component carriers (CCs). Each CC has a number of physical resource blocks (PRBs), where the PRB is the smallest unit of allocation. Each cell in the system is comprised of an enhanced NodeB (eNB) that serves a number of U user equipments (Ues) randomly dropped in the layout (including LTE-A UEs and Rel-8 UEs). 


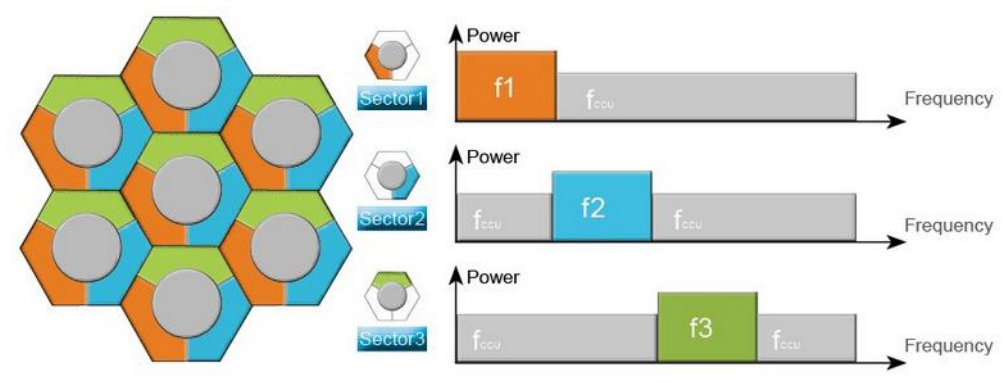

Figure 1. Soft frequency reuse technique [10]

\section{LTE-A NETWORK PLANNING AND DIMENSIONING}

Coverage planning and traffic dimensioning are initial step in the process of LTE-A network planning considering propagation model for hilly area [12]. This process results in the form of estimates of the number of cells $(\mathrm{N})$ required to meet the planning area within certain parameters of coverage and minimum signal level, regardless of the aspect of capacity. Planning consists of evaluation coverage link budget downlink and uplink. Maximum path loss is calculated based on the level of CINR required by the receiver, then converted to the eNB cell radius, using propagation models that correspond to the design area. Radio Link Budget is the component that characterizes the design of coverage [13]. By using the parameters of LTE-A, frequency band, and the clutter digital map of experimental observation area, we then do the calculations to find the cell radius. This value is derived using an empirical equation in the form of propagation Cost-231 expression.

$$
P L=46+33.9 \log (f)-13.83 \log h_{T}-a\left(h_{R}\right)+\left(44.9-6.55 \log h_{T}\right) \log d+C
$$

where,

$$
\begin{aligned}
& C=\left\{\begin{array}{l}
0, \text { for sub-urbanarea } \\
3, \text { for urbanarea }
\end{array}\right. \\
& a\left(h_{R}\right)=\left\{\begin{array}{l}
(1.1 \log (f)-0.7) h_{R}-(1.56 \log (f)-0.8), \text { for small } / \text { mediumcity } \\
3.2\left(\log 11.75 h_{R}\right) 2-1.1, \text { for } l \arg \text { e city }
\end{array}\right.
\end{aligned}
$$

Given the frequency used in the planning of the network is $1800 \mathrm{MHz}$, as a result we are able to demonstrate that the required number of eNB in our design is shown in Table 1 . We can se that the required numbers of eNB are 38 for urban and sub-urban area.

Now we consider that those numbers of eNB is going to be plot above the digital map inside the observation area with the help of network simulation tool. As a result we can see in the Figure 2, the deployment of 38 eNB covering both urban and sub-urban environment. In the figure, the black line is used to distinguish the boundary of urban and sub-urban areas in the tested area. The overall location site based on position of longitute-latitute coordinate and also altitute are recorded in network tool and is used in the simulation to find the performance of power partition SFR method. Another perspective of LTE-A network planning is determination of parameters in traffic and service model that is used in order to maximize throughput to be achieved by users. In this regard, the throughput means a minimum data rate that a single user can achieve. Throughput $(S)$ calculation for each session is expressed as,

$$
S=\frac{T_{p} x S_{D R} x B_{R}}{1-B L E R}
$$

where $T_{p}$ is throughput per session kbps, $S_{D R}$ denotes PPPsession time in second, $B_{R}$ denotes bearer rate in $\mathrm{kbps}$, and BLER is block error rate. The model parameters and traffic services selection will determine a single user throughput. Therefore, single user throughput can be calculated using,

Table 1. The calculated required number of cell (eNB). 


\begin{tabular}{lll}
\hline \multirow{2}{*}{ Parameter } & \multicolumn{2}{c}{ Type of Observed Area } \\
\cline { 2 - 3 } & Urban & Sub-urban \\
\hline Radius, d [km] & 1,39 & 1,8 \\
Cell area, [km2] & 3,76 & 6,41 \\
Total observed area, $[\mathrm{km} 2]$ & 104,84 & 62,83 \\
Cell site number & 28 & 10 \\
\hline
\end{tabular}

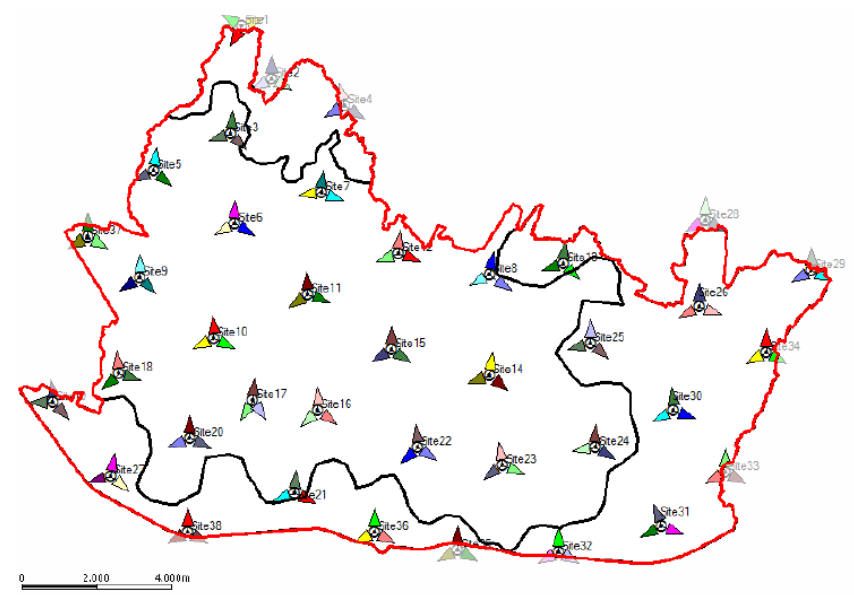

Figure 2. eNB deployment on the observation area.

$$
h_{s}=\sum\left[\frac{S \times B H S A \times R\left(1+P_{A R}\right)}{3600}\right] k b p s
$$

where $h_{s}$ is a single user throughput IP, BHSA denotes busy hour session attempt, $R$ is penetration rate and $P_{A R}$ denotes peak to average ratio. Total network throughput is a single user throughput $\left(h_{s}\right) \mathrm{x}$ total targetted user. Once traffic parameter and service model are considered in the network planning, the total network throughput can be determined using Equation (4) and (5). Depending on how traffic parameter and service modeled, we are able to estimate network capacity and that the required number of eNB is also predicted. However, in this work number of eNB required to cover service area is determined from the coverage requirement rather than capacity estimation.

\section{INTER-CELL INTERFERENCE COORDINATION METHOD IN SFR}

Now let's consider that SFR will be employed in the simulated network environment derived in Section 3. We devide each cell into multiple area which means we employ a multilevel SFR technique. Similar to SFR-2 (2 level SFR), multilevel SFR (ML-SFR) also defines a network-level framework for resource allocation in each cell refer to power partition for each eNB. Generally, a UE can be assigned the resources in the frequency band that covers it. However, we suggest allocating resources to a UE in the bands with the possible smallest coverage to optimize the system performances. As mentioned above in Section 2, we can assign $\alpha$ in the network that produce the smallest interference so that the performance of for example CINR is increased. Discussing about $\alpha$, which is the ratio between cell center user power and cell edge user power, we pointed out that when $\alpha$ increases, the cell edge capacity decreases and the cell center capacity increases, and vise versa. Regarding all the traffic happens at the cell edge only or center only, $\alpha$ should be 0 or 1 . However, when the traffic is uniformly distributed, the optimal value for $\alpha$ is still an open problem. Implementation of SFR into the deployed network may raise the problem of power allocation algorithm and the collision probability between each co-channel carrier. Analytically, collision probability directly affecting the interference reduction factor $\left(f_{I C I C-D L}\right)$ and can be expressed as,

$$
f_{I C I C-D L}^{T X_{i}(i c)-T X_{j}(j c)}=10 \log \left(P \frac{T X_{i}(i c)-T X_{j}(j c)}{\text { collision }}\right)
$$


Parameter of interference reduction factor is required to determine the amount of each resource element interference energy. It is used to perform calculations of CINR through the following Equation.

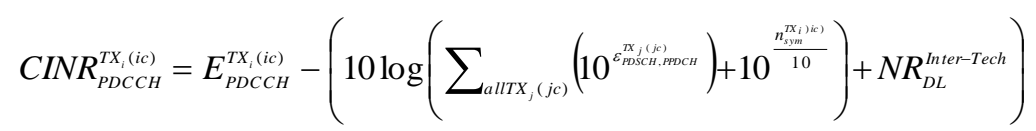

Besides CINR, we also perform computation of the downlink throughput. Throughput that is considered in this paper is the peak RLC Channel throughput on the downlink direction which can be expressed by,

$$
C T P_{P-D L}^{M_{i}}=\frac{R_{D L}^{T X_{i}(i c)} x \eta_{B_{D L}^{M_{i}}}}{D_{\text {frame }}} x \frac{N_{F B-C E}^{T X_{i}(i c)}}{N_{F B}^{T X_{i}(i c)}}
$$

where $R_{D L}^{T X_{i}(i c)}$ is the total downlink resources in the cell, $\eta_{B_{D L}^{u_{i}}}$ is barrier efficiency (bit per symbol), $D_{f r a m e}$ is the frame duration (10 ms based on 3GPP standard), $N_{F B}^{T X_{i}(i c)}$ is the number of resource block, and $N_{F B-C E}^{T X_{i}(i c)}$ denotes the number of alocated resources block to the cell edge. In this work we also observe block error rate (BLER) which is formulated by,

$$
C T P_{E-D L}^{M_{i}}=C T P_{P-D L}^{M_{i}} x\left(1-B L E R\left(B_{D L}^{M_{i}}\right)\right)
$$

In case of SFR implementation in the developed network area, we first calculate the ratio of the transmission power as described in Section 2. So the calculation of the value of $\alpha, \mathrm{P}_{\mathrm{CEU}}$, and $\mathrm{P}_{\mathrm{CCU}}$ can be obtained through the Equations (1) and (2) are the result indicated by the Table 1. The power difference between the end of the cell and the center of the cell causes the difference in the value of Maximum Allowable Path Loss, so it can be known how the edge radius ratio and the cell middle radius ratio. To obtain radius ratio of the two condition we can use the following expression.

$$
L_{s a, d_{\max }}=L_{s a, d i}+10 m \log _{10}\left(\frac{d_{i, \max }}{d_{i}}\right)
$$

where $L_{s a, d_{\max }}-L_{s a, d i}=1 / \alpha \quad$ therefore,

$$
\frac{1}{\alpha}=10 m \log _{10}\left(\frac{d_{i, \max }}{d_{i}}\right)
$$

Table 2. The Calculation of The Value of $\alpha$ and the Transmission Power.

\begin{tabular}{ccccc}
\hline$\alpha$ & $\mathrm{P}_{\mathrm{CCU}}[\mathrm{dBm}]$ & $\mathrm{P}_{\mathrm{CEU}}[\mathrm{dBm}]$ & $\begin{array}{c}\text { Power } \\
\text { Difference }[\mathrm{dB}]\end{array}$ & $\mathrm{d}_{\mathrm{i}} / \mathrm{d}_{\mathrm{i}, \max }$ \\
\hline 0.0 & 0.0 & 47.8 & 47.8 & 0.0 \\
0.1 & 37.0 & 47.0 & 10.0 & 0.69 \\
0.2 & 39.3 & 46.3 & 7.0 & 0.83 \\
0.3 & 40.5 & 45.7 & 5.2 & 0.88 \\
0.4 & 41.3 & 45.2 & 3.9 & 0.91 \\
0.5 & 41.8 & 44.8 & 3.0 & 0.92 \\
0.6 & 42.1 & 44.4 & 2.2 & 0.94 \\
0.7 & 42.4 & 44.0 & 1.5 & 0.95 \\
0.8 & 42.7 & 43.6 & 0.9 & 0.96 \\
0.9 & 42.8 & 43.3 & 0.5 & 0.96 \\
1.0 & 43.0 & 43.0 & 0.0 & 0.96 \\
\hline
\end{tabular}



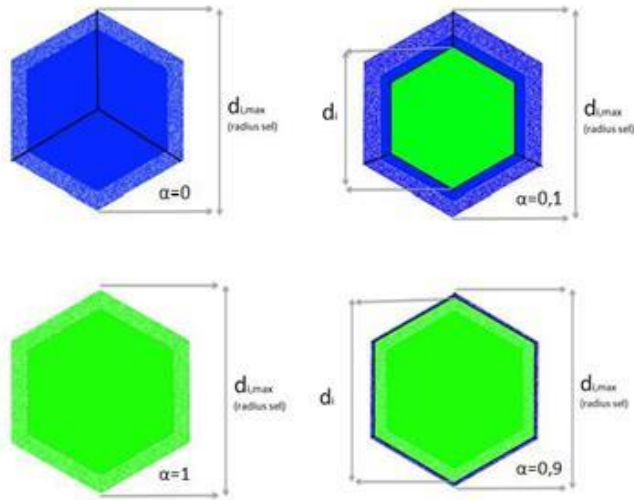

Figure 3. Comparison of the cell radius with the SFR radius.

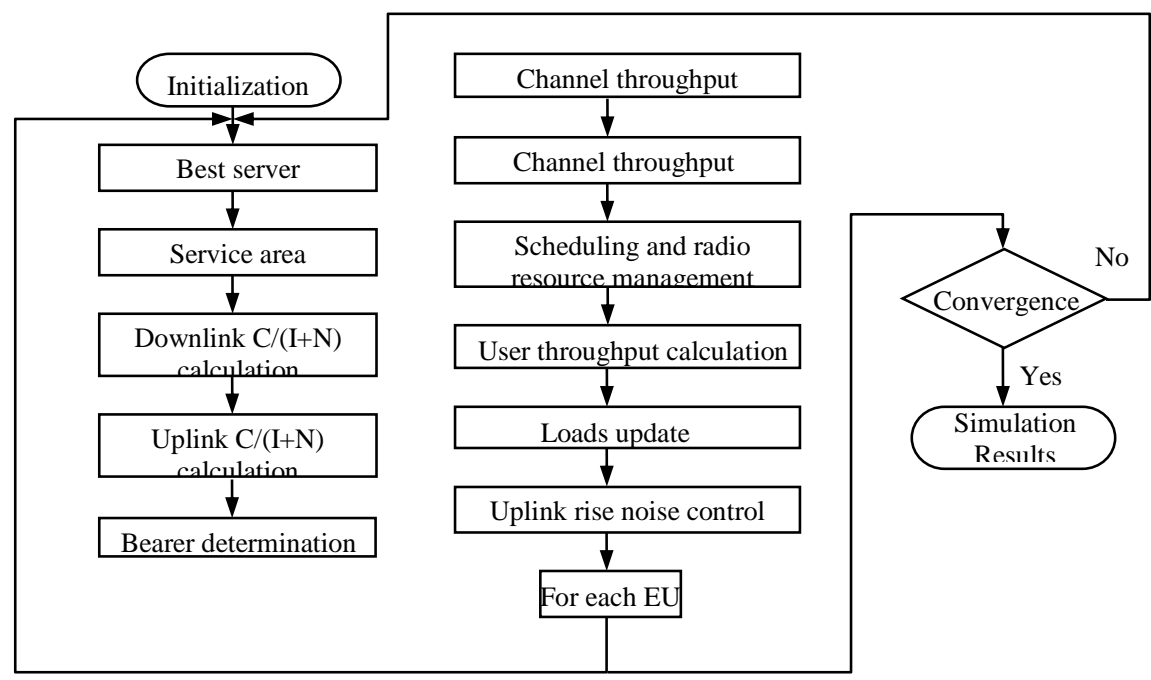

Figure 4. Monte carlo simulation algorithm.

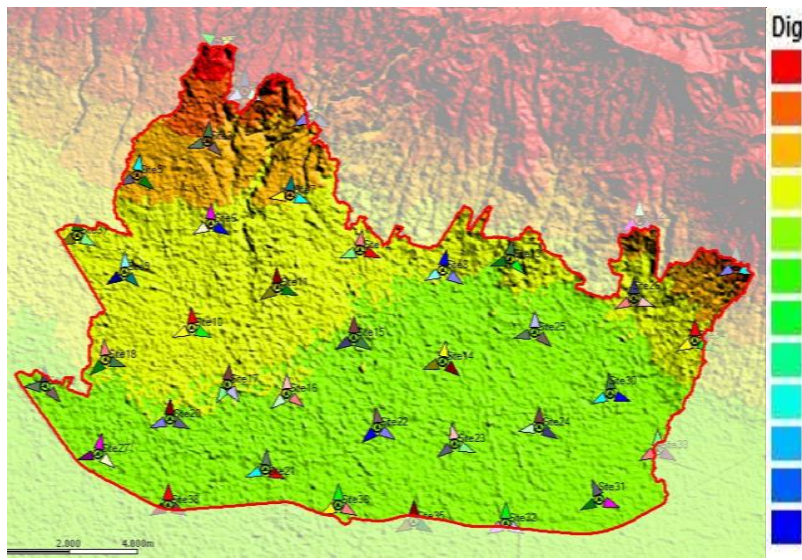

Digital Terrain Model

Altitude $(\mathrm{m})>=1,000$

$900<=$ Altitude $(\mathrm{m})<1.000$

$800<=$ Altitude $(\mathrm{m})<900$

$700<=$ Altitude $(\mathrm{m})<800$

$600<=$ Altitude $(\mathrm{m})<700$

$500<=$ Altitude $(\mathrm{m})<600$

$400<=$ Altitude $(\mathrm{m})<500$

$300<=$ Altitude $(\mathrm{m})<400$

$200<=$ Altitude $(\mathrm{m})<300$

$100<=$ Altitude $(\mathrm{m})<200$

$0<=$ Altitude $(\mathrm{m})<100$

Altitude $(m)<0$

Figure 5. Experimental service area topology

where $m$ is a path loss exponent, $L_{s a, d i}$ is an MAPL (maximum allowable path loss), and $L_{s a, d m a x}$ denotes an MPAL for the power transmit of $d_{i, \max }$. The comparison $\boldsymbol{d}_{\boldsymbol{i}}$ and $\boldsymbol{d}_{\boldsymbol{i} \text {,max }}$ is illustrated in Figure 3 and its value is also shown in Table 2. The blue color indicates FR3 while the green color is FR1. And a third outer cell area 
with the color texture of the cell edge. The value of $\alpha=0$ to $\alpha=1$ shows the slowly changing from FR1 to FR3, and therefore this scheme is called the Soft Frequency Reuse.

\section{SIMULATION RESULT AND DISCUSSIONS}

Simulation is then carried out as an approach method to evaluate the proposed multiple-SFR in LTE-A network. We use monte carlo simulation over which it is able to describe the influence of different services to the density of traffic on the customers who are not homogeneous. Monte carlo algorithm in our case can be describe as described in Figure 4.

The first step is to generate user location according to the user traffic map. In the process of generating the user location, we assume that the possibility of user connection is based on the parameter that contained in the traffic map for the type of services such as BHCA or the number of sessions in one hour ( $\mathrm{N}$ sesion), the traffic volume (in Kbyte) of VDL and VUL, and the good service downlink throughput (TPDL) and uplink (TPUL). The next step is determining the best server and service area which mean that the decisive sector of the cell where each user location based on the strength of the signal. We then calculate the CINR of the downlink and uplink experienced by each user. After CINR is obtained, to each user, it is determined the bearer that used based on the threshold of CINR of MCS. Having obtained the type of bearer we calculate the total throughput in the network.

\subsection{Effect of SFR to the CINR}

Now we can obtained the CINR inside the network service area following the digital map that is used in our experiment. Figure 5 describes the digital map based on elevated map of our experimental service area to evaluate the performance of inter-cell interference mitigation of SFR implementation. CINR at every point of location inside the map is simulated and evaluated for each value of $\alpha$. As for example, CINR for $\alpha=0, \alpha=0.1$, and $\alpha=1$ are depicted in the Figure 6. The changing in the value of CINR is caused by the amount of interference power that occurs randomly. For $\alpha=0.1$, CINR on the cell edge is the highest CINR among other $\alpha$ because it has the highest $\mathrm{P}_{\mathrm{CEU}}$ and it used FR3 scenario in the whole area of its cell edge. Average CINR of the whole cells is observed remain constant in each value $\alpha$ when the CINR value at the cell edge is quite high. This condition indicates a decrease CINR at the cell center caused by small Pccu based on the allocation of transmission power. Therefore, it makes the condition that means more susceptible to interference.

\subsection{Effect of SFR into BLER}

Another results in this experiment is BLER which is a parameter that states the number of block packet error due to inter-cell interference of SFR implementation. BLER is obtained from the results of monte carlo simulation in which we simulated for each user who is trying to access to LTE-A network. The lower the BLER the better the performance of the system. If the BLER is too high, it means there will be too many errors in data transmission received causing the data becomes invalid. Table 3 shows BLER for each value of $\alpha$. From the simulation result, average BLER for FR1 scheme in the entire cell is $4.33 \%$ while in the cell edge is $15 \%$ which means that the performance is worst and exceed the value of tolerated value. With the implementation of SFR, that the value of $\alpha=0.9$, BLER at the cell edge is able to be lower than $7.93 \%$ or nearly half of the BLER on FR1 scheme without SFR. BLER will continuously decrease with the smaller value of $\alpha$. At each decreasing $\alpha$ by 0.1, BLER is able to become $0.5 \%-2 \%$ decreasing. We observe when $\alpha=0.1$, BLER is $2.79 \%$ or decreasing by $12.21 \%$ from FR 1 scheme. Decreasing value of BLER on the cell edge means that when $\alpha$ is small, the interference is also small. If we look at the Table 2, when $\alpha=0.6$ the BLER value in the entire cell is almost equal to the BLER value on the cell edge which ranges in value of 4.7\%. It indicates the amount of errors that occur in the cell edge and the cell center is almost equal to the average error that occurred on the whole cells. This is to show that when $\alpha=0.6$, an overall network performance in the same cell is the sama as there is no gap between the performance at the cell center or in the cell edge. 


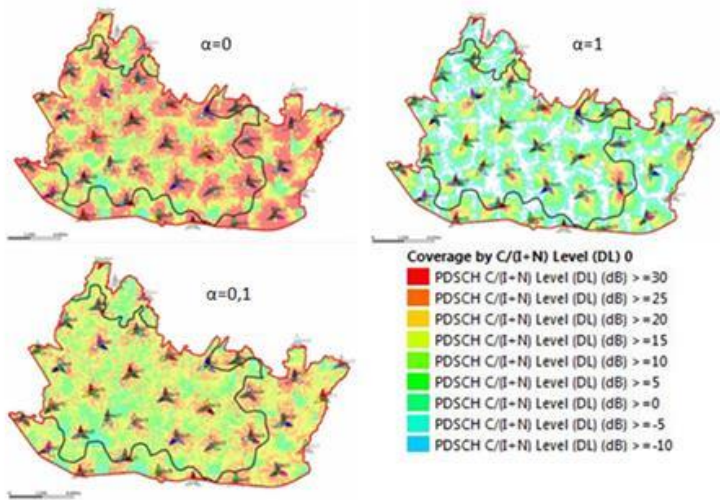

Figure 6. Simulated CINR.

Table 3. BLER for each $\alpha$.

\begin{tabular}{lcc}
\hline \multicolumn{1}{c}{$\alpha$} & $\begin{array}{c}\text { Average BLER } \\
\text { for the whole service area }\end{array}$ & $\begin{array}{c}\text { Average BLER } \\
\text { at the cell edge }\end{array}$ \\
\hline$\alpha=0$ (FR3) & $6,61 \%$ & $0,94 \%$ \\
$\alpha=0,1$ & $5,67 \%$ & $2,79 \%$ \\
$\alpha=0,2$ & $5,34 \%$ & $3,16 \%$ \\
$\alpha=0,3$ & $5,19 \%$ & $3,62 \%$ \\
$\alpha=0,4$ & $5,15 \%$ & $3,81 \%$ \\
$\alpha=0,5$ & $4,98 \%$ & $4,30 \%$ \\
$\alpha=0,6$ & $4,74 \%$ & $4,71 \%$ \\
$\alpha=0,7$ & $4,63 \%$ & $4,89 \%$ \\
$\alpha=0,8$ & $4,58 \%$ & $5,76 \%$ \\
$\alpha=0,9$ & $4,44 \%$ & $7,93 \%$ \\
$\alpha=1$ (FR1) & $4,33 \%$ & $15,00 \%$ \\
\hline
\end{tabular}

\subsection{Effect of SFR into Throughput}

Throughput is an important parameter in evaluationg the performance of SFR implementation in LTE-A network. Based on our simulation, it can be observed the variation of $\alpha$ would have a big impact to the network or individual UE throughput. Table 3 shows the relation of $\alpha$ with the average throughput in the whole network and average throughput in the cell edge. When $\alpha=0$, the average throughput in the whole cells is equal to the average throughput at the cell edge that is $15.5 \mathrm{Mbps}$. This small throughput value caused by the bandwidth used for each sector is only $1 / 3$ of the total bandwidth. By the time the value of $\alpha$ is increased, which means the average throughput is also increased, but the average throughput at the cell edge decrease. It means that interference have occurred. When the value of $\alpha=1$, throughput at the cell edge decreases to only 4 Mbps.

Figure 7 shows the decreasing value of the cell throughput and increase in throughput at the cell edge for each $\alpha$ value in the FR1 scheme. For detail, Table 4 exmplains that the value of $\alpha=0.9$ with a decrease in the average throughput to only by $0.87 \%$ but the cell edge throughput increased by nearly $40 \%$. The highest interval occurs when the value of $\alpha=0.5$ as the reduction in average throughput of $6.11 \%$ an increase in throughput at the cell edge by almost $60 \%$ of throughput at the cell edge FR1. On the other hand, it indicates a decrease in the average throughput at the cell center.

\subsection{Effect of SFR into the Throughput of Each Traffic Load}

To determine the overall performance of SFR, then simulation ranging from low to high traffic load. Traffic load is here defined as the proportion of Physical Resource Block (PRB). Therefore, when only $10 \%$ PRB diunakan in a cell, then the traffic load is $10 \%$, whereas if the entire PRB has used the traffic load has a maximum or $100 \%$.

Table 4. Throughput for each $\alpha$.

\begin{tabular}{ccc}
\hline$\alpha$ & $\begin{array}{c}\text { Average Throughput } \\
\text { for the whole service area }\end{array}$ & $\begin{array}{c}\text { Average Throughput } \\
\text { at the cell edge }\end{array}$ \\
\hline$\alpha=0$ (FR3) & $15.512,91$ & $15.512,91$ \\
$\alpha=0,1$ & $24.059,01$ & 13.163 \\
$\alpha=0,2$ & $26.264,34$ & 12.242 \\
\hline
\end{tabular}




\begin{tabular}{ccc}
\hline$\alpha=0,3$ & $27.737,36$ & 11.576 \\
$\alpha=0,4$ & $28.629,66$ & 11.059 \\
$\alpha=0,5$ & $29.210,74$ & 10.579 \\
$\alpha=0,6$ & $29.583,80$ & 10.158 \\
$\alpha=0,7$ & $30.051,27$ & 9.670 \\
$\alpha=0,8$ & $30.438,90$ & 9.178 \\
$\alpha=0,9$ & $30.840,04$ & 7.145 \\
$\alpha=1$ (FR 1$)$ & $31.111,29$ & 4.299 \\
\hline
\end{tabular}
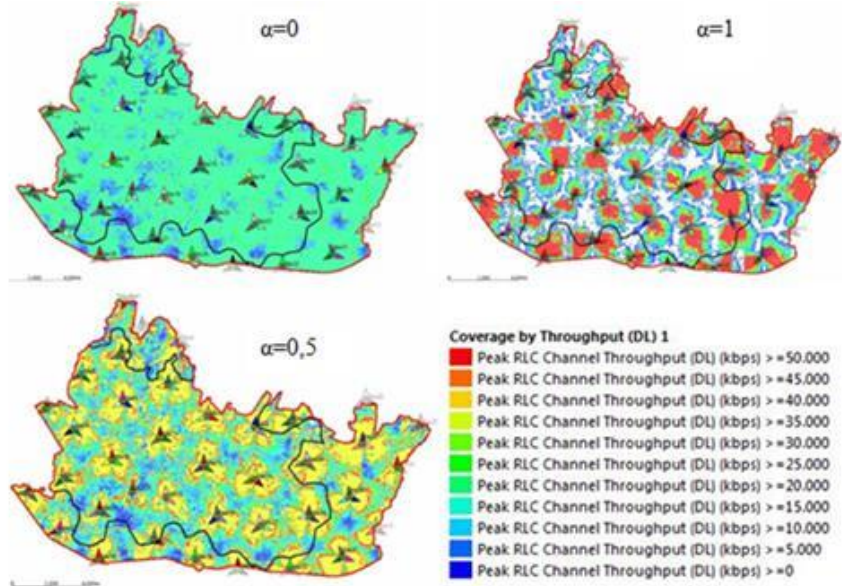

Coverage by Throughput (DU) Peak RLC Channel Throughput (DU) (abps) > $=50.000$

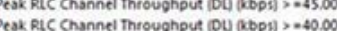
Peak RLC Channel Throughout (DU) abpal) $>3.35000$ Peask RIC Channel Throughput (DL) (abps) $>=30.000$ Peak RLC Channei Throughput (DLI) (xbDs) $>=25.000$ Peak RLC Channel throughput (DU) (Kops) $>=20.000$ Peak RiC Channel throughput (DU) (Rbps) $>=15.000$ Peak RLC Channel Throughput (DC) (xbDs) > $=10.000$

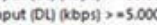
Peak RIC Channel Throughout (DC) (xbos) > $>0$
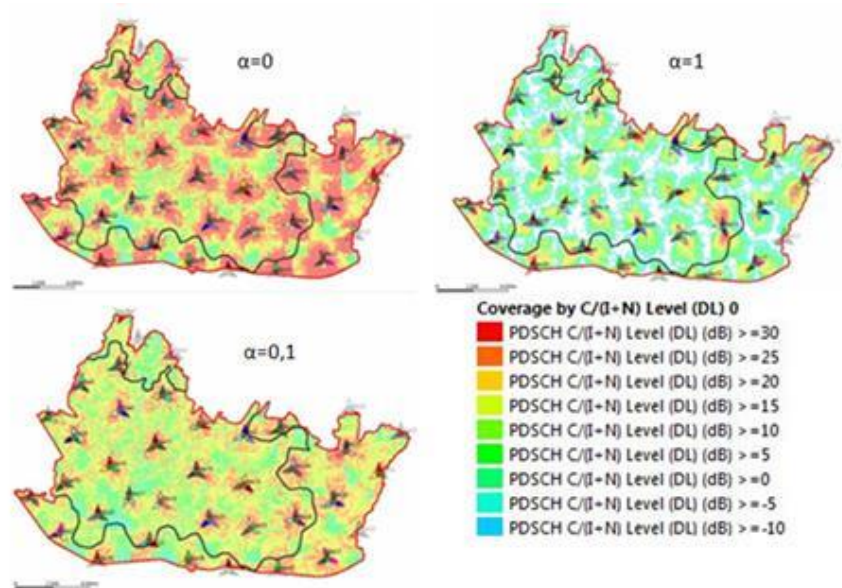

Coverage by $\mathrm{C} / \mathrm{Q}+\mathrm{N})$ Level $(\mathrm{DL}) \mathrm{O}$ PDSCH C/(I-N) Level (DU) (dB) > $>30$ PDSCH C/(I-N) Level (DL) (dB) $>=25$ PDSCH C/ $(\mathrm{D}-\mathrm{N}$ ) Level (DU) $(\mathrm{AB})>=20$

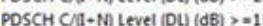
POSCH CA $(M)$ Level (DL) (AB) >-15 POSCH $C D$ -

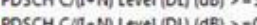
POSCH C(I-N) Level (DL) (AE) > $>=0$ POSCH CIIN $N$ ) Level (DL) (dB) > >.-5 POSCH COR-N) Level (DU) (dB) $>=-10$

Figure 7. Simulated throughput at different $\alpha$.

Figure 8 shows a graph of the average throughput values for each percentage of the traffic load. It seems clear that the scheme FR1 and SFR much better than FR3 scheme. FR3 scheme certainly has an average throughput is small because of the bandwidth allocated to each sector is only $1 / 3$ of the total bandwidth owned. With FR1 scheme, the average throughput at the time of the full traffic load is 31.11 Mbps, whereas when the traffic load is only $10 \%$ yield $21.33 \mathrm{Mbps}$ throughput, lower than the throughput at SFR scheme $\alpha=0.4$, namely $21.73 \mathrm{Mbps}$. Based on the graph of the simulation results can also be observed that when the traffic load is low, SFR scheme has better throughput than FR1, especially SFR with a value of $\alpha=0.8$. But then began the traffic load of $50 \%$ up to a maximum traffic load, FR 1 scheme is able to produce better throughput. The higher the traffic load in the neighboring cells, means more subcarriers are used, so the higher the resulting interference. In accordance with the results of this simulation can be concluded that the scheme SFR is able to overcome the interference in the cell at the time of low traffic and SFR scheme is less able to influence the interference that occurs when the full traffic load.

The highest throughput is owned by FR3 scheme, either at the time minimum traffic load to the maximum, which ranges in value of $11.9 \mathrm{Mbps}$ to $15.5 \mathrm{Mbps}$. While the scheme FR1 lowest throughput among other scheme which is at a value of $2.3 \mathrm{Mbps}$ to $4.2 \mathrm{Mbps}$. 


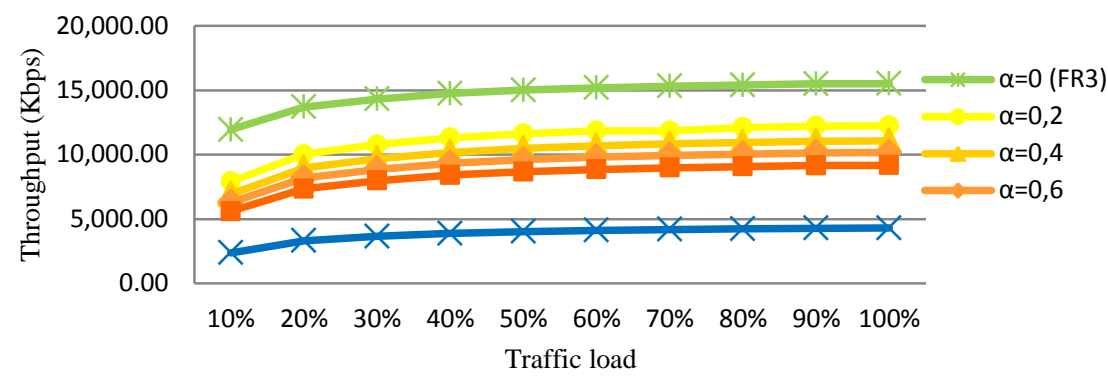

Figure 8. Throughput at the cell edge in each value of traffic load.

\section{CONCLUSIONS}

Multilevel SFR as an inter-cell interference management technique in LTE-A network has been presented. LTE-A network deployment that has been simulated in this work produce a cell coverage of about 99.9\%. Multilevel or power partition of SFR scheme has proven its ability to improve network performance at the cell edge in terms of a parameter value such CINR, BLER and throughput. With this technique, the highest average CINR at the cell edge is $10.5 \mathrm{~dB}$ when $\alpha$ equals 0.1 or increase more than four times from FR1 technique. Average BLER at the cell edge is able to reduced up to $2.75 \%$, namely when $\alpha=0.1$ or decrease about $12.21 \%$ from FR1 technique. Multiple SFR also shows a balance performance in case of average BLER on the cell edge, cell center, and the entire cell which is equal to $4.7 \%$. Additionally, when the traffic load is lower than 50\%, average throughput that can be achieved with multilevel SFR technique in the whole cell is better than that of FR1 and FR3 throughput performance. Finally, multilevel SFR technique is capable of producing an average throughput at the cell edge better than FR1 either at low or high traffic load. The highest throughput is obtained when $\alpha=0.1$ which is about $13,16 \mathrm{Mbps}$ or an increase of twice the FR1 scheme.

\section{REFERENCES}

[1] K. Odeyemi and E. Ogunti, "Capacity Enhancement for High Data Rate Wireless Communication System," International Journal of Electrical and Computer Engineering (IJECE), vol 4, no. 5, pp. 800-809, October 2014.

[2] G. Giambene, V.A. Le, T. Bourgeau, and H. Chaouchi, "Soft frequency reuse schemes for heterogeneous LTE systems," in Proceeding of IEEE International Conference on Communications (ICC), June 2015.

[3] M. Qian, et.al., "Adaptive Soft Frequency Reuse Scheme for Wireless Cellular Networks," IEEE Transactions on Vehicular Technology, vol. 64, no. 1, pp. 118-131, 2015.

[4] X. Shui, M. Zhao, P. Dong, and J. Kong, "A novel dynamic soft frequency reuse combined with power reallocation in LTE uplinks," in Proceeding of International Conference on Wireless Communications and Signal Processing (WCSP 2012), October 2012.

[5] W. Pramudito and A. Alsusa, "Confederation Based RRM with Proportional Fairness for Soft Frequency Reuse LTE Networks," IEEE Transactions on Wireless Communications, vol. 13, no. 3, pp. 1703-1715, 2014.

[6] Y. Yang, L. Chen, P. Zhao, and W. Wang, "Adaptive Power Ratio Updating Algorithm in Soft Frequency Reuse Scheme," in Proceeding of IEEE 78th Vehicular Technology Conference (VTC Fall), September 2013.

[7] P. Zhang, Y. Mo, and B. Wang, "Dynamic soft frequency reuse based on sector factor for LTE downlink transmission," in Proceeding of International Conference on Wireless Communications and Signal Processing, October 2013

[8] X. Yang, "A Multilevel Soft Frequency Reuse Technique for Wireless Communication Systems," IEEE communications letters, vol. 18, no. 11, pp. 1983-1986, November 2014.

[9] Y. Yu, et. all, "Performance Analysis of Soft Frequency Reuse for Inter-cell Interference Coordination in LTE Networks", $10^{\text {th }}$ International Symposium on Communications and Information Technologies, pp. 505-509, October 2010.

[10] ZTE Document, "Enhanced ICIC for LTE-A HetNet," 2011.

[11] F. John O and O.Y. O, "Radio Frequency Propagation Mechanisms and Empirical Models for Hilly Areas," International Journal of Electrical and Computer Engineering (IJECE), vol 3, no. 3, pp. 372-376, June 2013.

[12] E. Dahlman, S. Parkvall, and J. Skold. 4G: LTE/LTE-Advanced for Mobile Broadband. Elsevier, second edition, 2014.

[13] A.R. Mishra. Fundamentals of Cellular Network Planning and Optimization 2G/2.5G/3G Evolution to 4G, John Wiley \& Sons, 2004. 